\title{
A very simple introduction to Bayesian statistics: From coin flips to insight
}

Daniel M. Zuckerman

zuckermd@ohsu.edu

Department of Biomedical Engineering

Oregon Health \& Science University

\begin{abstract}
Bayesian statistical analyses are a growing part of the chemical and biological sciences for several reasons. Most importantly, the Bayesian approach of predicting underlying models based on data corresponds naturally with examination of complex systems, whether using wet-lab or computational means. The Bayesian structure also provides a systematic basis for estimating uncertainty in model parameters and permits incorporation of prior information in a quantitative and consistent way. While easy to state in words, these strengths of Bayesian analysis can be difficult to assimilate for beginners. This short article presents essential Bayesian concepts using very simple examples and the absolute minimum mathematics needed to maintain rigor.
\end{abstract}

\section{Introduction}

Bayesian statistics now permeate every area of science, and with good reason. The approach enables us to see and think beyond a mean and standard deviation in qualitatively new ways. Most notably, Bayesian analysis enable quantitative conclusions about unseen, underlying processes (rather than just properties of the data itself) and the approach also enables quantitative use of prior information. In biology, Bayesian analysis is critical for hidden Markov modeling of cellular processes visualized via livecell microscopy [Bronson-2009, Persson-2013] and plays a growing role in analysis of molecular simulation data [Mostofian-2019], to give just two examples. The early, heated controversy about Bayesian analysis is a distant memory that can be evoked through the advocacy writing by physicist E.T. Jaynes [Jaynes-1976].

Although there are plenty of introductions to Bayesian statistics, many of these implicitly assume mathematical sophistication. One that I like is geared toward biophysics [Hines-2015], but for nonexperts the emphasis on 'conjugate priors' diminishes the impact. An introduction more in the spirit of the present write-up is [Eddy-2004].

Here, I'd like to introduce Bayesian thinking in perhaps the simplest way possible, but with rigor. We want to understand the point of it, and get a better grip on those mysterious priors. This article is adapted from a blog post of mine [Zuckerman-2018]. 


\section{Examples and Discussion}

We'll look at the classic example of coin flipping. Say we flipped a coin three times and got the sequence HHT (heads-heads-tails). Was our coin fair? Was the probability of heads, $p(H)$, exactly half? And how confident can we be about our answer?

First notice that the question, "Was our coin fair?," inquires about the underlying system itself, not the data we got. This is a key point ...

Key Observation 1: Bayesian analysis attempts to characterize the underlying model or distribution, rather than characterizing the observed data. Of course, the data is used to characterize the model, as we will see. If our Bayesian analysis really can tell us about all possible $p(H)$ values, then we can assess whether we should believe the value $1 / 2$ or $2 / 3$ or whether we really can't tell the difference. To start on this process, let's define the probability of heads by the symbol $q=p(H)$. Because there are only two possible values ( $\mathrm{H}$ or $\mathrm{T}), q$ fully characterizes the underlying distribution, which we'll call the "model".

The goal of Bayesian analysis is to estimate the conditional probability of any model (any $q$ value) given the particular data (HHT) that was obtained, a probability denoted by $p$ (model $\mid$ data). We may also incorporate prior information about the model. The function $p$ (model | data) is called the posterior distribution because it is obtained after the data.

In our case, the posterior distribution we want is $p(q \mid \mathrm{HHT})$, an estimate of relative probabilities for different $q$ values given the sequence of flips $\mathrm{HHT}$. If we know the probability of each $q$, not only can we examine the special value $q=1 / 2$ but, more importantly, we can see how $q=1 / 2$ compares to $q=2 / 3$ or any other value of interest.

Bayesian analysis can be seen a simple application (though confusing to the beginner!) of elementary probability theory, so let's start with a common sense approach. One simple thing that we can do is assume a model (a $q$ value) and then calculate the probability of our data (HHT) in that model. To do this we don't even need to assign a numerical $q$ value. We can use elementary probability theory: the product of a series of events is simply the product of the individual probabilities. Thus, we have $p(\mathrm{HHT} \mid q)=$ $q^{2}(1-q)$, which is true for any $q$. In other words, we have an explicit function for the probability of the data given a model embodied in $q$.

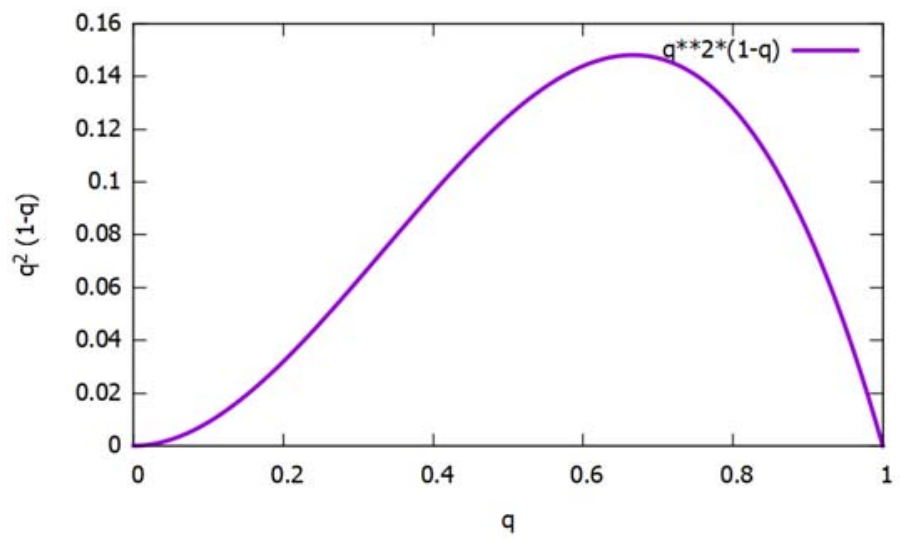

The function $p(\mathrm{HHT} \mid q)$ is plotted here and looks like what we would expect, with a peak at $q=2 / 3$. That is, no other $q$ value yields higher probability for the sequence of flips HHT. Moreover, there are other features that make intuitive sense: the probability to observe HHT given $q$ values near zero or one are 
vanishingly small. All the preceding comments apply explicitly to the probability of seeing the data given the model embodied in the parameter $q$. Recall that $q$ determines the probabilities of all events.

And yet, all the observations could be "turned around" and qualitatively, they also apply to the posterior distribution we're seeking. The posterior is the probability of $q$ given the data, $p(q \mid \mathrm{HHT})$. Clearly the most likely $q$ (in the absence of any other information) must be $2 / 3$. And since we have seen both $\mathrm{H}$ and $T$ values in our data, the probability that $q=0$ or 1 must vanish. So we already seem to be on the right track. At least in our case, we can draw a tentative conclusion ...

Key Observation 2: Qualitatively, $p$ ( model | data $) \sim p$ ( data | model ). That is, it seems we can use easy-to-calculate data probabilities $p$ (data $\mid$ model ) to estimate model probabilities. This point will be formalized below.

We are finally ready to jump into the formal math of the Bayesian approach, which should be a bit easier to appreciate with the HHT example in mind. The Bayesian framework requires us only to understand the following rule of probability for a two-dimensional probability distribution:

$$
p(x, y)=p(y \mid x) p(x)=p(x \mid y) p(y)
$$

Here $x$ and $y$ are any variables of interest (continuous or discrete-valued like $\mathrm{H}$ and $\mathrm{T}$ ), $p(x, y)$ is the joint distribution over both variables, meaning that $p d x d y$ is the probability in a $d x d y$ square for a continuous distribution or - in a discrete system $p$ is the absolute probability. Since $p(x, y)$ is a two-dimensional distribution it must be normalized when integrated (or summed) over all $x$ and $y$ values. Also, we can integrate (sum) over all $y$ values for each $x$ to get the "marginal" distribution $p(x)$, or vice versa to get the other marginal $p(y)$. See any basic probability reference for more information on these issues.

The Bayesian picture employs the two-dimensional joint distribution of data and models. This is the key conceptual leap, assuming model and data probabilities can be quantified together in the first place. It sounds a bit strange at first, but because models can be defined via their parameters, mathematically a regular probability distribution is still being used. We can therefore refine our first observation.

Key Observation 1a. The Bayesian picture assumes that there is a true mathematical distribution of models (not just data), and not all models are equally likely.

This is a rather abstract point, and perhaps you may question it. After all, logically, it seems that there must have been only a single model that generated our data - we just don't know what it was. True, but that single model is assumed to be unknowable (with absolute certainty). Instead, from the statistical perspective, we instead hope only to characterize the likelihood of different underlying models based on the information we have.

Back to coin flipping. To make progress, we simply need to manipulate the rules for two-dimensional probability distributions discussed above in order to get the posterior distribution for $q$ we want. We write

$$
p(q \mid \mathrm{HHT})=\frac{p(\mathrm{HHT} \mid q) p(q)}{p(\mathrm{HHT})}
$$

which is called Bayes' rule or formula. Here, the posterior of interest is on the left. On the right are factors that may be familiar or not. The factor $p(\mathrm{HHT} \mid q)$ is the simplest - it's the probability of HHT given a specific $q$ value, as we already discussed. The factor $p$ (HHT) technically is the overall probability of $\mathrm{HHT}$ in all possible models, but the nice thing about it is that it does not depend on $q$ at all: it's a constant for any $q$ value on the left, so we can think of it as an uninteresting normalization constant.

Most intriguing here is the so-called "prior" distribution of $q$ values, $p(q)$. This factor represents our knowledge about which $q$ values are likely - independent of the data. For instance, if our HHT data was 
generated by a real physical coin, perhaps it's much harder to make a coin exhibiting very small or large $q$ values (near 0 or 1 ), so those probabilities might be smaller. Perhaps it's your big brother using his magic set with unfair coins and you know that he only has coins with the values $q=0.3$ and 0.5 .

Let's look more closely at the example of your big brother, who's tried to fool you lots of times - and so you know he's $90 \%$ likely to choose the unfair coin $(q=0.3)$ and only $10 \%$ likely to choose the fair coin. This is the prior information we will assume. In that case, given just two coin flips to make things even simpler, we can generate the following table listing all the joint and marginal probabilities.

\begin{tabular}{|c|c|c|c|c|c|}
\hline & HH & HT & TH & TT & $\begin{array}{c}\text { Marginal: } \\
\mathbf{p}(\mathbf{q})\end{array}$ \\
\hline $\mathbf{q}=\mathbf{0 . 3}$ & $\begin{array}{c}p(\mathrm{H}) p(\mathrm{H}) p(q=0.3) \\
=0.3^{\star} 0.3^{\star} 0.9\end{array}$ & $\begin{array}{c}p(\mathrm{H}) p(\mathrm{~T}) p(q=0.3) \\
=0.3^{*} 0.7^{*} 0.9\end{array}$ & $0.7^{\star} 0.3^{\star} 0.9$ & $\begin{array}{c}0.7^{\star} 0.7^{\star} 0.9 \\
=0.441\end{array}$ & 0.9 \\
\hline $\mathbf{q}=\mathbf{0 . 5}$ & $0.5^{\star} 0.5^{\star} 0.1$ & $0.5^{\star} 0.5^{\star} 0.1$ & $0.5^{\star} 0.5^{\star} 0.1$ & $0.5^{\star} 0.5^{\star} 0.1$ & 0.1 \\
\hline $\begin{array}{c}\text { Marginal } \\
\text { (sum) }\end{array}$ & 0.106 & 0.214 & 0.214 & 0.466 & \\
\hline
\end{tabular}

This table is worth staring at for a while. The eight entries in the middle of the table are the posterior probabilities for each possible set of coin tosses and $q$ value. The entries have been normalized so that all eight sum to 1 . The marginal $p(q)$ in the right column is the prior.

Try picking a given data value (e.g., HH or TT) and see what the estimated $q$ probabilities are. This will tell you both the best guess for $q$ given any particular data, as well as the relative chance you're wrong, given the assumed prior. If the two flips were TT, for example, then the table shows there is a $95 \%$ likelihood $(0.441 / 0.466)$ that $q=0.3$. The key thing here, which is subtle, is that $95 \%$ is different than the 90\% prior: the data have helped point you toward the more likely outcome. (Other pairs of flips make $q=$ 0.3 less likely than its prior, as you can check.) More data will help a lot, not surprisingly, as described below.

Let's build on Bayes' rule to re-examine the case where $q$ can take on any value between 0 and 1 . The posterior, our estimate for the likelihood of $q$, is given by $p(q \mid$ HHT $) \propto p$ (HHT $\mid q) p(q)$, where we have omitted the unimportant constant factor $p(\mathrm{HHT})$. The relation is almost what we hypothesized before from common sense: the probability of the model given the data is proportional to the probability of the data given the model aside from prior information we may have to correct that. But in hindsight, it's also clear that prior information about possible or likely $q$ values must correct our estimate. Thus, we can make another observation.

Key Observation 3: Bayes' formula tells us, mathematically, how to incorporate "prior" knowledge about the set of (im)possible models. The formula makes intuitive sense, in that the intrinsic, data-independent prior probability of any model acts as a corrective pre-factor or weight for the overall "posterior" estimate of a model's probability. If we have no prior information about models, then the posterior model probability is simply proportional to the data probability - e.g., $p$ (HHT $\mid q)$.

Let's consider whether and when the prior really matters. Perhaps you've heard the choice of prior is extremely important - or maybe you've heard the prior doesn't really matter. Well, both are true in different situations.

We need to look more closely at the data probability, $p(\mathrm{HHT} \mid q)=q^{2}(1-q)$. This is just the product of the probabilities for each event, so more generally, we have

$$
p\left(x_{1}, x_{2}, x_{3}, \ldots, x_{N} \mid \text { model }\right)=p\left(x_{1}\right) p\left(x_{2}\right) p\left(x_{3}\right) \cdots p\left(x_{N}\right)
$$


where each factor on the right side really depends on the model $p\left(x_{i}\right)=p\left(x_{i} \mid\right.$ model $)$. If each event is a coin toss and there are many of them, then in contrast to the $q^{2}(1-q)$ function shown above, the full product of probabilities will become a very sharply peaked function of $q$. (You should check this for a sequence containing some arbitrary large numbers of $\mathrm{H}$ and $\mathrm{T}$ values.) Thus, in the case of large $\mathrm{N}$, really only a small range of $q$ values will be probable. If the peak in $q$ is sharp enough, just a tiny range will be probable regardless of the prior. So we can add to our list of observations.

Key Observation 4: The prior distribution (assumption about models) is more important when there is less data and decreasingly important as the amount of data grows.

Maybe your brain is getting tired, even in this briefest of introductions, but there is one more key point about Bayesian statistics.

One of the most important features of the Bayesian formulation is that it inherently brings with it the ability to characterize the range of uncertainty associated with a certain model. In other words, it's easy to find the most likely $q$ value (which was $2 / 3$ for our HHT example), but our original question was whether we think the coin was fair or not.

So what do we think ... was the coin fair?!? Of course it's impossible to answer yes or no based on the data alone, but we can make a simple calculation to quantify the situation.

Let's take the simple case of a constant prior for now (same probability for all $q$ values), in which case the posterior distribution of $q$ is $p(q \mid \mathrm{HHT}) \propto q^{2}(1-q)$. Since we know the distribution for any $q$ value, we can simply evaluate it at the two values of interest. We find

$$
\frac{p\left(q=\frac{1}{2} \mid \text { HHT }\right)}{p\left(q=\frac{2}{3} \mid \text { HHT }\right)} \approx 0.84
$$

That is, with a constant prior, the likelihood that $\mathrm{HHT}$ data was generated by a fair coin $(q=1 / 2)$ is $84 \%$ as high as the simple guess $q=2 / 3$. There's quite a good chance the coin was fair. But as your common sense would tell you, we can't be sure from just three coin tosses. Nevertheless, the Bayesian formulation lets us quantify different possibilities based on the data seen.

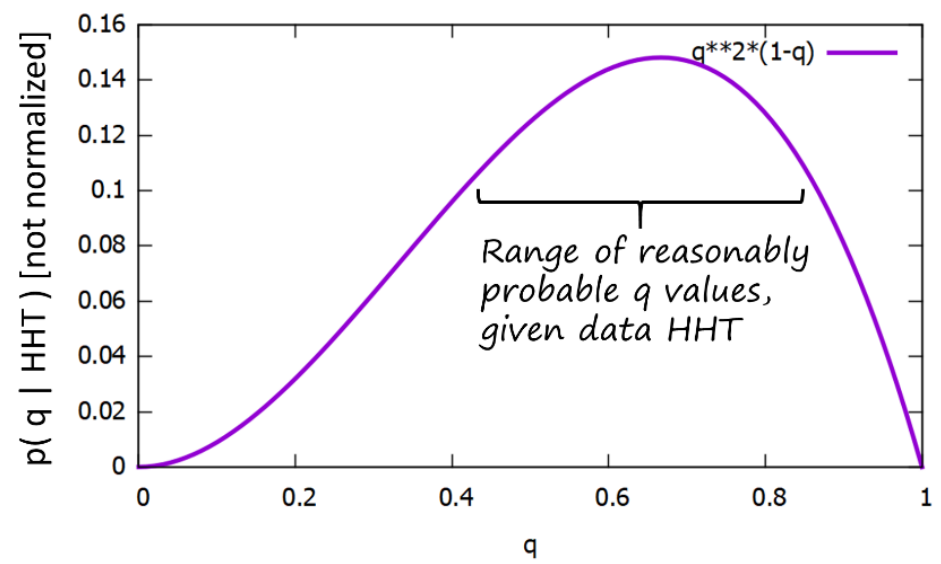

You can take this type of analysis further by determining a range of reasonably probable $q$ values as sketched above. What range you want to use will depend on what question you are trying to answer. Quantifying a range, however, is a topic beyond the scope of this post. In any case, we can make our final observation. 
Key Observation 5: The Bayesian formulation intrinsically quantifies uncertainty in the underlying model. This is because the posterior distribution of models provides an estimate for the likelihood of all models which thus can be compared directly.

Note that standard confidence intervals from frequentist statistics are not direct characterizations of the underlying model in the same way that a Bayesian analysis. Rather, frequentist analysis characterizes ranges of outcomes. To take an extreme case, if you know a coin can be heads or tails but with an unknown $q$ value - and only a single coin flip is performed yielding $\mathrm{H}$ - frequentist statistics really cannot characterize anything about the situation whereas Bayesian analysis can.

\section{Acknowledgements}

The author is grateful for support from the National Science Foundation under grant MCB 1715823.

\section{References}

[Bronson-2009] Bronson, J. E., Fei, J., Hofman, J. M., Gonzalez Jr, R. L., \& Wiggins, C. H. (2009). Learning rates and states from biophysical time series: a Bayesian approach to model selection and single-molecule FRET data. Biophysical journal, 97(12), 3196-3205.

[Eddy-2004] Eddy, S. R. (2004). What is Bayesian statistics?. Nature biotechnology, 22(9), 1177-1178.

[Hines-2015] Hines, K. E. (2015). A primer on Bayesian inference for biophysical systems. Biophysical journal, 108(9), 2103-2113.

[Jaynes-1976] Jaynes, E. T. (1976). Confidence intervals vs Bayesian intervals. In Foundations of probability theory, statistical inference, and statistical theories of science, Harper and Hooker, Eds. (pp. 175-257). Springer, Dordrecht.

[Mostofian-2019] Mostofian, B., \& Zuckerman, D. M. (2019). Statistical uncertainty analysis for smallsample, high log-variance data: Cautions for bootstrapping and Bayesian bootstrapping. Journal of chemical theory and computation, 15(6), 3499-3509.

[Persson-2013] Persson, F., Lindén, M., Unoson, C., \& Elf, J. (2013). Extracting intracellular diffusive states and transition rates from single-molecule tracking data. Nature methods, 10(3), 265-269.

[Zuckerman-2018] Zuckerman, D. M. (2018). Absolutely the simplest introduction to Bayesian statistics. http://statisticalbiophysicsblog.org/?p=233 